\title{
Testing CPT symmetry with CMB measurements
}

\author{
J.-Q. Xia ${ }^{1}$, H. Li ${ }^{2}$, X. Wang ${ }^{3}$, and X. Zhang ${ }^{1}$ \\ 1 Institute of High Energy Physics, Chinese Academy of Science, PO Box 918-4, Beijing 100049, PR China \\ e-mail: xiajq@mail.ihep.ac.cn \\ 2 Department of Astronomy, School of Physics, Peking University, Beijing 100871, PR China \\ 3 Fakultät für Physik, Universität Bielefeld, 33615 Bielefeld, Germany
}

Received 17 January 2008 / Accepted 27 February 2008

\section{ABSTRACT}

\begin{abstract}
Aims. We study the possibility of testing Charge-Parity-Time Reversal symmetry (CPT) with cosmic microwave background (CMB) measurements.

Methods. Working with an effective lagrangian of the photon with CPT violation $\mathcal{L} \sim p_{\mu} A_{v} \tilde{F}^{\mu v}$, which causes the polarization vectors of the propagating CMB photons to be rotated, we determine the rotation angle $\Delta \alpha$ using the BOOMERanG 2003 and the WMAP3 angular power spectra.

Results. In this analysis, we have included the newly-released TC and GC $(l<450)$ information of WMAP3 and found $\Delta \alpha=$ $-6.2 \pm 3.8$ deg at a $68 \%$ confidence level.

Conclusions. This result slightly increases the significance for the CPT violation obtained in our previous paper (Feng et al. 2006) $\Delta \alpha=-6.0 \pm 4.0 \mathrm{deg}(1 \sigma)$. Furthermore, we examine the constraint on the rotation angle from the simulated polarization data with Planck precision. Our results show that the future Planck measurement will be sensitive to $\Delta \alpha$ at the level of 0.057 deg and will be able to test the CPT symmetry with a higher precision.
\end{abstract}

Key words. cosmology: theory - cosmology: cosmic microwave background - cosmology: cosmological parameters

\section{Introduction}

In the standard model of particle physics, CPT is a fundamental symmetry. Probing its violation is an important way to search for the new physics beyond the standard model. Up to now, CPT symmetry has passed a number of high-precision experimental tests and no definite signal of its violation has been observed in the laboratory. So, the present CPT violating effects, if they exist, should be very small to be amenable to the experimental limits.

The CPT symmetry could be dynamically violated in the expanding universe (Li et al. 2007). The cosmological CPT violation mechanism investigated in the literature ( $\mathrm{Li}$ et al. 2002; Li \& Zhang 2003; Li et al. 2004; Feng et al. 2005; Li et al. 2007) has an interesting feature, which is that the CPT violating effects at present are too small to be detected by the laboratory experiments, but large enough in the early universe to account for the generation of matter-antimatter asymmetry (Li et al. 2002; Li \& Zhang 2003; Li et al. 2004, 2007). More importantly, these type of CPT violating effects could be accumulated to be observable in the cosmological experiments (Feng et al. 2005; Li et al. 2007; Feng et al. 2006). With the accumulation of high-quality observational data, especially those from the CMB experiments, cosmological observation becomes a powerful way to test the CPT symmetry.

Here we study the CMB polarizations and CPT violation in the photon sector with an effective Lagrangian (Carroll et al. 1990; Carroll \& Field 1991):

$\mathcal{L}=-\frac{1}{4} F_{\mu \nu} F^{\mu v}+\mathcal{L}_{\mathrm{cs}}$, where $\mathcal{L}_{\mathrm{cs}} \sim p_{\mu} A_{\nu} \tilde{F}^{\mu \nu}$ is a Chern-Simons term, $p_{\mu}$ is an external vector, and $\tilde{F}^{\mu \nu}=(1 / 2) \epsilon^{\mu \nu \rho \sigma} F_{\rho \sigma}$ is the dual of the electromagnetic tensor. This Lagrangian is not gauge invariant, but the action is gauge independent if $\partial_{\nu} p_{\mu}=\partial_{\mu} p_{\nu}$. This may be possible if $p_{\mu}$ is constant in spacetime or the gradient of a scalar field in the quintessential baryo-/leptogenesis (Li \& Zhang 2003; Li et al. 2002; De Felice et al. 2003) or the gradient of a function of the Ricci scalar in gravitational baryo-/leptogenesis (Li et al. 2004; Davoudiasl et al. 2004). The Chern-Simons term violates Lorentz and CPT symmetries when the background value of $p_{\mu}$ is nonzero.

One of the physical consequences of the Chern-Simons term is the rotation of the polarization direction of electromagnetic waves propagating over large distances (Carroll et al. 1990). From the Lagrangian (1), we can directly obtain the equation of motion for the electromagnetic field

$\nabla_{\mu}\left(\nabla^{\mu} A^{v}-\nabla^{v} A^{\mu}\right)=-p_{\mu} \epsilon^{\mu \nu \rho \sigma}\left(\nabla_{\rho} A_{\sigma}-\nabla_{\sigma} A_{\rho}\right)$.

After imposing Lorentz gauge condition $\nabla_{\mu} A^{\mu}=0$, it becomes

$\nabla_{\mu} \nabla^{\mu} A^{v}+R_{\mu}^{v} A^{\mu}=-p_{\mu} \epsilon^{\mu \nu \rho \sigma}\left(\nabla_{\rho} A_{\sigma}-\nabla_{\sigma} A_{\rho}\right)$

where $R_{\mu}^{v}$ is the Ricci tensor. With the geometric optics approximation, the solution to the equation of motion is expected to be: $A^{\mu}=\operatorname{Re}\left[\left(a^{\mu}+\epsilon b^{\mu}+\epsilon^{2} c^{\mu}+\ldots\right) \mathrm{e}^{i S / \epsilon}\right]$, where $\epsilon$ is a small number. With this ansatz, one can easily see that the Lorentz gauge condition implies $k_{\mu} a^{\mu}=0$, where the wave vector $k_{\mu} \equiv \nabla_{\mu} S$ is orthogonal to the surfaces of constant phase and represents the direction along which photons travel. The vector $a^{\mu}$ is the product of a scalar amplitude $A$ and a normalized polarization vector $\varepsilon^{\mu}, a^{\mu}=A \varepsilon^{\mu}$, with $\varepsilon_{\mu} \varepsilon^{\mu}=1$. Hence in the Lorentz gauge, the wave vector $k_{\mu}$ is orthogonal to the polarization vector $\varepsilon^{\mu}$. 
Substituting this solution into the modified Maxwell Eq. (3) and neglecting the Ricci tensor, the leading order of $\epsilon$ the equation is $k_{\mu} k^{\mu}=0$. This indicates that photons still propagate along the null geodesics. The effect of Chern-Simons term appears at the next order, $k^{\mu} \nabla_{\mu} \varepsilon^{v}=-p_{\mu} \epsilon^{\mu \nu \rho \sigma} k_{\rho} \varepsilon_{\sigma}$. We can see that the ChernSimons term makes $k^{\mu} \nabla_{\mu} \varepsilon^{v}$ not vanished. This means that the polarization vector $\varepsilon^{v}$ is not transported parallel to the light-ray. The vector rotates as the photon propagates in spacetime.

We consider here the spacetime described by spatially-flat Friedmann-Robertson-Walker (FRW) metric. The null geodesics equation is $\left(k^{0}\right)^{2}-k^{i} k^{i}=0$. We assume that photons propagate along the positive direction of $x$ axis, i.e., $k^{\mu}=\left(k^{0}, k^{1}, 0,0\right)$ and $k^{1}=k^{0}$. Gauge invariance guarantees that the polarization vector of the photon has only two independent components that are orthogonal to the propagating direction. So, we are only interested in the changes of the components of the polarization vector, $\varepsilon^{2}$ and $\varepsilon^{3}$. Assuming $p_{\mu}=p_{0}$ to be a non-vanishing constant, we obtain the following equations: $\mathrm{d} \varepsilon^{2} / \mathrm{d} \lambda+\mathcal{H} k^{0} \varepsilon^{2}=p_{0} k^{0} \varepsilon^{3}$, $\mathrm{d} \varepsilon^{3} / \mathrm{d} \lambda+\mathcal{H} k^{0} \varepsilon^{3}=-p_{0} k^{0} \varepsilon^{2}$, where we have defined the affine parameter $\lambda$, which measures the distance along the light-ray $k^{\mu} \equiv \mathrm{d} x^{\mu} / \mathrm{d} \lambda$, and the reduced expansion rate $\mathcal{H} \equiv \dot{a} / a$. The polarization angle is defined as $\chi \equiv \arctan \left(\varepsilon^{3} / \varepsilon^{2}\right)$. The rotation angle is given by

$\Delta \chi \equiv \chi_{0}-\chi_{z}=-\int_{\eta_{z}}^{\eta_{0}} p_{0} \mathrm{~d} \eta=\int_{t_{0}}^{t_{z}} p_{0} \frac{\mathrm{d} t}{a}$,

where the subscript $z$ is the redshift of the source when the light was emitted. For CMB photons, the source is the last scattering surface with $z \simeq 1100^{1}$. The subscript 0 indicates the present time. As we know, a vector rotated by an angle $\Delta \chi$ in a fixedcoordinates frame is equivalent to a fixed vector observed in a coordinates frame that is rotated by $-\Delta \chi$. So, with the notion of coordinates frame rotation, the rotation angle is

$\Delta \alpha=-\Delta \chi=\int_{t_{z}}^{t_{0}} p_{0} \frac{\mathrm{d} t}{a}=p_{0} r_{z}$

with $r_{z}$ being the comoving distance of the light source away from us. This phenomena is known as "cosmological birefringence". This rotation angle $\Delta \alpha$ can be obtained by observing polarized radiation from distant sources such as radio galaxies, quasars, and CMB.

The Stokes parameters $Q$ and $U$ of the CMB polarization can be decomposed into a gradient-like $(\mathrm{G})$ and a curl-like $(\mathrm{C})$ component (Kamionkowski et al. 1997). For the standard theory of CMB, the TC and GC cross-correlation power spectra vanish. With the existence of cosmological birefringence, the polarization vector of each photon is rotated by an angle $\Delta \alpha$. Hence one would observe nonzero TC and GC correlations, even if they are zero at the last scattering surface. Denoting the rotated quantities

\footnotetext{
${ }^{1}$ Besides the CMB photons which come from the last scattering surface, we could have observed the different $\mathrm{CMB}$ photons that traveled different distances as well. However, we are only interested in linear perturbations of $\mathrm{CMB}$ photons in this paper. $\mathrm{CMB}$ polarizations are already linear phenomena. They are not existent at the zeroth order. When calculating the variations of polarizations due to CPT violation in the perturbation theory up to linear order, we may ignore the fluctuations of the traveling distances of CMB photons. Otherwise, these fluctuations combined with polarizations would give higher order results, that are beyond the scope of this paper. So, we only consider linear perturbations and we can assume that each CMB photon detected by us travelled the same distance.
}

with a prime, one gets (Feng et al. 2005; Lue et al. 1999):

$C_{1}^{\prime \mathrm{TC}}=C_{1}^{\mathrm{TG}} \sin (2 \Delta \alpha)$,

$C_{1}^{\prime} \mathrm{GC}=\frac{1}{2}\left(C_{1}^{\mathrm{GG}}-C_{1}^{\mathrm{CC}}\right) \sin (4 \Delta \alpha)$,

$C_{1}^{\prime \mathrm{TG}}=C_{1}^{\mathrm{TG}} \cos (2 \Delta \alpha)$,

$C_{1}^{\prime \mathrm{GG}}=C_{1}^{\mathrm{GG}} \cos ^{2}(2 \Delta \alpha)+C_{1}^{\mathrm{CC}} \sin ^{2}(2 \Delta \alpha)$,

$C_{1}^{\prime \mathrm{CC}}=C_{1}^{\mathrm{CC}} \cos ^{2}(2 \Delta \alpha)+C_{1}^{\mathrm{GG}} \sin ^{2}(2 \Delta \alpha)$,

while the temperature power-spectrum TT remains unchanged.

\section{Method and results}

In our study, we analyze globally to the CMB data with the public available Markov Chain Monte Carlo package CosmoMC ${ }^{2}$ (Lewis \& Bridle 2002), which has been modified to allow the rotation of the power spectra discussed above, with a new free parameter $\Delta \alpha$. We assume the purely adiabatic initial conditions and impose the flatness condition motivated by inflation. Our most general parameter space is: $\boldsymbol{P} \equiv$ $\left(\omega_{\mathrm{b}}, \omega_{\mathrm{c}}, \Theta_{\mathrm{s}}, \tau, n_{\mathrm{s}}, \log \left[10^{10} A_{\mathrm{s}}\right], r, \Delta \alpha\right)$, where $\omega_{\mathrm{b}} \equiv \Omega_{\mathrm{b}} h^{2}$ and $\omega_{\mathrm{c}} \equiv$ $\Omega_{\mathrm{c}} h^{2}$ are the physical baryon and cold dark matter densities relative to the critical density, $\Theta_{\mathrm{s}}$ is the ratio of the sound horizon to the angular diameter distance at decoupling, $\tau$ is the optical depth to re-ionization, $A_{\mathrm{s}}$ and $n_{\mathrm{s}}$ characterize the primordial scalar power spectrum, $r$ is the tensor to scalar ratio of the primordial spectrum. For the pivot of the primordial spectrum, we set $k_{\mathrm{s} 0}=0.05 \mathrm{Mpc}^{-1}$. In our calculation we have assumed that the cosmic rotation angle is not too large and imposed a conservative flat prior $-\pi / 2 \leq \Delta \alpha \leq \pi / 2$.

In our calculation, we combine the full data of Three-year Wilkinson Microwave Anisotropy Probe (WMAP3) (Spergel et al. 2007; Page et al. 2007; Hinshaw et al. 2007; Jarosik et al. 2007) including the information of TC and GC power spectra $(l<450)$, and Balloon Observations of Millimetric Extragalactic Radiation and Geophysics (BOOMERanG 2003, B03) (Jones et al. 2006; Piacentini et al. 2006; Montroy et al. 2006). We calculate the likelihood of TT, TG, GG, and CC power spectra using the routine for computing the likelihood supplied by the WMAP team. As for the TC and GC power-spectra data $(l<450)$, which are just the preliminary suboptimal results currently, we simply assume the Gaussian likelihood function ${ }^{3}$

$$
\begin{aligned}
& \mathcal{L}_{\mathrm{TC} / \mathrm{GC}}=\exp \left(-\frac{\chi_{\mathrm{TC} / \mathrm{GC}}^{2}}{2}\right), \\
& \chi_{\mathrm{TC} / \mathrm{GC}}^{2}=\sum_{1}\left(\frac{C_{1, \mathrm{th} / \mathrm{GC}}^{\mathrm{TC}}-C_{1, \mathrm{obs}}^{\mathrm{TC} / \mathrm{GC}}}{\sigma_{1}}\right)^{2},
\end{aligned}
$$

where $C_{1, \text { th }}^{\mathrm{TC} / \mathrm{GC}}$ and $C_{1, \mathrm{obs}}^{\mathrm{TC} / \mathrm{GC}}$ denote the theoretical value and the experimental data of the TC or GC power spectra of WMAP3, $\sigma_{1}$ denotes the variance of estimated power spectra at each multi-pole.

In the computation of the CMB spectra, we have considered lensing contributions. Furthermore, we make use of the

\footnotetext{
2 http://cosmologist.info/

3 We are very grateful to Professor Eiichiro Komatsu (the member of WMAP group) for the email communications on the use of the TC and GC data of WMAP3. Because the current released TC and GC information of WMAP3 are not very accurate and do not include the correlations between different $l$ values, our simple Gaussian likelihood function Eq. (7) is accurate enough for the illustrative purpose.
} 


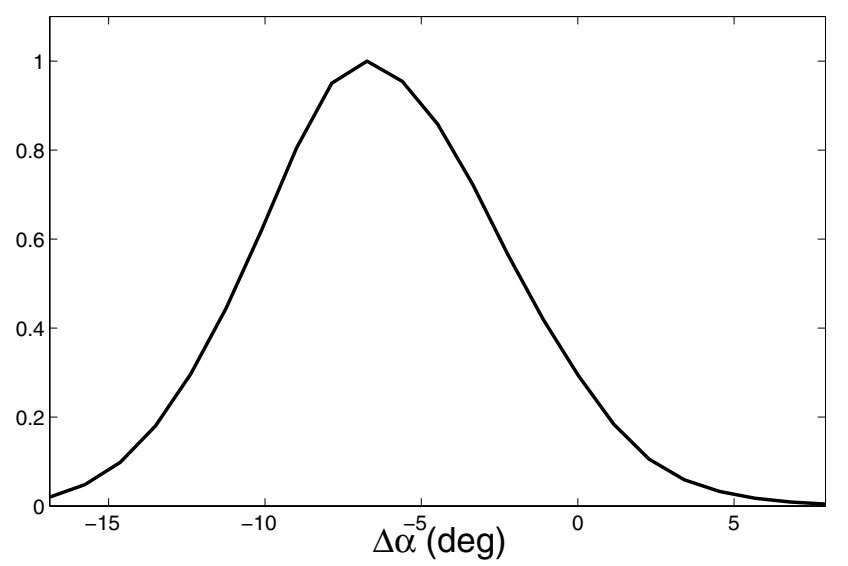

Fig. 1. One dimensional distribution on the rotation angle $\Delta \alpha$ from WMAP3 and B03 data.

Hubble Space Telescope (HST) measurement of the Hubble parameter $H_{0} \equiv 100 \mathrm{~h} \mathrm{~km} \mathrm{~s}^{-1} \mathrm{Mpc}^{-1}$ by multiplying a Gaussian likelihood function $h=0.72 \pm 0.08$ (Freedman et al. 2001). We also impose a weak Gaussian prior on the baryon density $\Omega_{\mathrm{b}} h^{2}=0.022 \pm 0.002(1 \sigma)$ from the big bang nucleosynthesis (Burles et al. 2001). Simultaneously, we will also use a cosmic age top-hat prior as $10 \mathrm{Gyr}<t_{0}<20 \mathrm{Gyr}$.

In Fig. 1, we plot our one-dimensional constraint on the rotation angle $\Delta \alpha$ from the combined WMAP3 and B03 data and find that the current $\mathrm{CMB}$ polarization data favor a nonzero rotation angle of the photons. The best fit value of the rotation angle is $\Delta \alpha=-0.122=-7.0 \mathrm{deg}$. Marginalizing the posterior distributions, we find that the mean value of the rotation angle is

$\Delta \alpha=-0.108 \pm 0.067=-6.2 \pm 3.8 \mathrm{deg}(1 \sigma)$,

which deviates the unrotated case $\Delta \alpha=0$ more than $1 \sigma$ and gives weak evidence for CPT violation. This result is frequency independent whereas the faraday rotation (Scannapieco \& Ferreira 1997), which also can give nonzero TC and GC power spectra, is frequency dependent and of the much smaller order than ours. On the other hand, currently several high-precision experimental tests have confirmed the CPT symmetry and do not detect apparent CPT violation in the laboratory. However, these two results are consistent. From Eqs. ((4), (5)) we find that the size of the effects of CPT violation on $\mathrm{CMB}$ polarization power spectra is an integrated effect enhanced during the propagation time, while at present the laboratory experiments only test the CPT symmetry. This means that the CPT violating effect should be too small to be detected at any specific time, and should be enhanced during the propagation time to be detectable by the CMB measurements.

In Fig. 2, we illustrate the effects of the rotation angle $\Delta \alpha$ on the power spectra for two cases $\Delta \alpha=0$ and $\Delta \alpha=-0.108$. The basic cosmological parameters we choose for these plots are the best fit values of global fitting: $\Omega_{b} h^{2}=0.0228, \Omega_{\mathrm{m}} h^{2}=$ $0.1320, \tau=0.099, H_{0}=73.1, n_{s}=0.965, A_{s}=2.2 \times 10^{-9}$ and $r=0.00866$. One can see from Fig. 2 that the $\mathrm{C}$-mode is very sensitive to the rotation angle $\Delta \alpha$. Direct measurements of the TC and GC power spectra are crucial and would give more stringent constraints than ones of other power spectra.

From the analysis of data points of the TC and GC power spectra of WMAP3 and B03, we find that this negative rotation angle is slightly preferred. First, in the B03 data, the TC power at $l \sim 250$ and $l \sim 350$ are both negative, whereas it is positive at $l \sim 450$. The GC power at $l \sim 250, l \sim 350$ and $l \sim 450$ are all negative. Based on the Eq. (6) and Fig. 2, we can see that the TC and GC power spectra of B03 really help to obtain this negative rotation angle. On the other hand, the quality of TC and GC power spectra of WMAP3 does not seem very good. The data points are distributed around the line $\Delta \alpha=0$ proportional and the error bars are large. Therefore, the WMAP3 polarization data can not contribute much to testing the CPT symmetry and does not improve the previous result of rotation angle significantly.

Feng et al. (2006) used the WMAP3 and B03 polarization data to constrain the rotation angle and found a similar result: $\Delta \alpha=-6.0 \pm 4.0 \mathrm{deg}$ at $1 \sigma$ confidence level. As we show above, the TC and GC information is very important for this type of analysis. In their analysis they did not include the TC and GC information of WMAP3 and set the TC and GC of power spectra of WMAP3 at zero, $C_{1}^{\mathrm{TC}}=0$ and $C_{1}^{\mathrm{GC}}=0$. This factor should affect the final result. Comparing these two results, we find that the mean value of our result moves away from zero and the error bar shrinks a little, which strengthens the conclusion on the CPT violation.

Moreover, Cabella et al. (2007) perform a wavelet analysis of the temperature and polarization maps of the $\mathrm{CMB}$ delivered by the WMAP3 experiment, which includes the information of TC and GC power spectra. They set a limit on the CMB photon rotation angle $\Delta \alpha=-2.5 \pm 3.0 \mathrm{deg}(1 \sigma)$. They found no evidence for the CPT violation from WMAP3 polarization data. However, in their analysis, they have not included the information of B03 polarization data. We also made an analysis without the B03 data and found that $\Delta \alpha=-1.6 \pm 10.3 \mathrm{deg}(1 \sigma)$, which means that the WMAP3 data only did not give any significant evidence for CPT violation. Therefore, we believe that they would obtain the similar result as ours if they also included the B03 polarization data in their analysis.

Obviously, the current CMB polarization data are not good enough to verify this possible CPT violation. We need more accurate CMB data, such as the Planck measurement ${ }^{4}$, in the near future. In Table 1, we list the assumed experimental specifications of the future Planck measurement. The likelihood function is $\mathcal{L} \propto \exp \left(-\chi_{\text {eff }}^{2} / 2\right)$ and

$\chi_{\mathrm{eff}}^{2}=\sum_{1}(2 l+1) f_{\mathrm{sky}}\left(\frac{A}{|\bar{C}|}+\ln \frac{|\bar{C}|}{|\hat{C}|}+3\right)$,

where $f_{\text {sky }}$ denotes the observed fraction of the sky in the real experiments, $A$ is defined as

$$
\begin{aligned}
A= & \hat{C}_{1}^{\mathrm{TT}}\left(\bar{C}_{1}^{\mathrm{GG}} \bar{C}_{1}^{\mathrm{CC}}-\left(\bar{C}_{1}^{\mathrm{GC}}\right)^{2}\right)+\hat{C}_{1}^{\mathrm{TG}}\left(\bar{C}_{1}^{\mathrm{TC}} \bar{C}_{1}^{\mathrm{GC}}-\bar{C}_{1}^{\mathrm{TG}} \bar{C}_{1}^{\mathrm{CC}}\right) \\
& +\hat{C}_{1}^{\mathrm{TC}}\left(\bar{C}_{1}^{\mathrm{TG}} \bar{C}_{1}^{\mathrm{GC}}-\bar{C}_{1}^{\mathrm{TC}} \bar{C}_{1}^{\mathrm{GG}}\right)+\hat{C}_{1}^{\mathrm{TG}}\left(\bar{C}_{1}^{\mathrm{TC}} \bar{C}_{1}^{\mathrm{GC}}-\bar{C}_{1}^{\mathrm{TG}} \bar{C}_{1}^{\mathrm{CC}}\right) \\
& +\hat{C}_{1}^{\mathrm{GG}}\left(\bar{C}_{1}^{\mathrm{TT}} \bar{C}_{1}^{\mathrm{CC}}-\left(\bar{C}_{1}^{\mathrm{TC}}\right)^{2}\right)+\hat{C}_{1}^{G C}\left(\bar{C}_{1}^{\mathrm{TG}} \bar{C}_{1}^{\mathrm{TC}}-\bar{C}_{1}^{\mathrm{TT}} \bar{C}_{1}^{\mathrm{GC}}\right) \\
& +\hat{C}_{1}^{\mathrm{TC}}\left(\bar{C}_{1}^{\mathrm{TG}} \bar{C}_{1}^{\mathrm{GC}}-\bar{C}_{1}^{\mathrm{GG}} \bar{C}_{1}^{\mathrm{TC}}\right)+\hat{C}_{1}^{\mathrm{GC}}\left(\bar{C}_{1}^{\mathrm{TG}} \bar{C}_{1}^{T C}-\bar{C}_{1}^{\mathrm{TT}} \bar{C}_{1}^{\mathrm{GC}}\right) \\
& +\hat{C}_{1}^{\mathrm{CC}}\left(\bar{C}_{1}^{\mathrm{TT}} \bar{C}_{1}^{\mathrm{GG}}-\left(\bar{C}_{1}^{\mathrm{TG}}\right)^{2}\right)
\end{aligned}
$$

and $|\bar{C}|$ and $|\hat{C}|$ denote the determinants of the theoretical and observed data covariance matrices respectively,

$$
\begin{aligned}
|\bar{C}|= & \bar{C}_{1}^{\mathrm{TT}} \bar{C}_{1}^{\mathrm{GG}} \bar{C}_{1}^{\mathrm{CC}}+2 \bar{C}_{1}^{\mathrm{TG}} \bar{C}_{1}^{\mathrm{TC}} \bar{C}_{1}^{\mathrm{GC}}-\bar{C}_{1}^{\mathrm{TT}}\left(\bar{C}_{1}^{\mathrm{GC}}\right)^{2} \\
& -\bar{C}_{1}^{\mathrm{GG}}\left(\bar{C}_{1}^{\mathrm{TC}}\right)^{2}-\bar{C}_{1}^{\mathrm{CC}}\left(\bar{C}_{1}^{\mathrm{TG}}\right)^{2}, \\
|\hat{C}|= & \hat{C}_{1}^{\mathrm{TT}} \hat{C}_{1}^{\mathrm{GG}} \hat{C}_{1}^{\mathrm{CC}}+2 \hat{C}_{1}^{\mathrm{TG}} \hat{C}_{1}^{\mathrm{TC}} \hat{C}_{1}^{\mathrm{GC}}-\hat{C}_{1}^{\mathrm{TT}}\left(\hat{C}_{1}^{\mathrm{GC}}\right)^{2} \\
& -\hat{C}_{1}^{\mathrm{GG}}\left(\hat{C}_{1}^{\mathrm{TC}}\right)^{2}-\hat{C}_{1}^{\mathrm{CC}}\left(\hat{C}_{1}^{\mathrm{TG}}\right)^{2} .
\end{aligned}
$$

\footnotetext{
4 http://sci.esa.int/science-e/www/area/index.cfm? fareaid=17/
} 

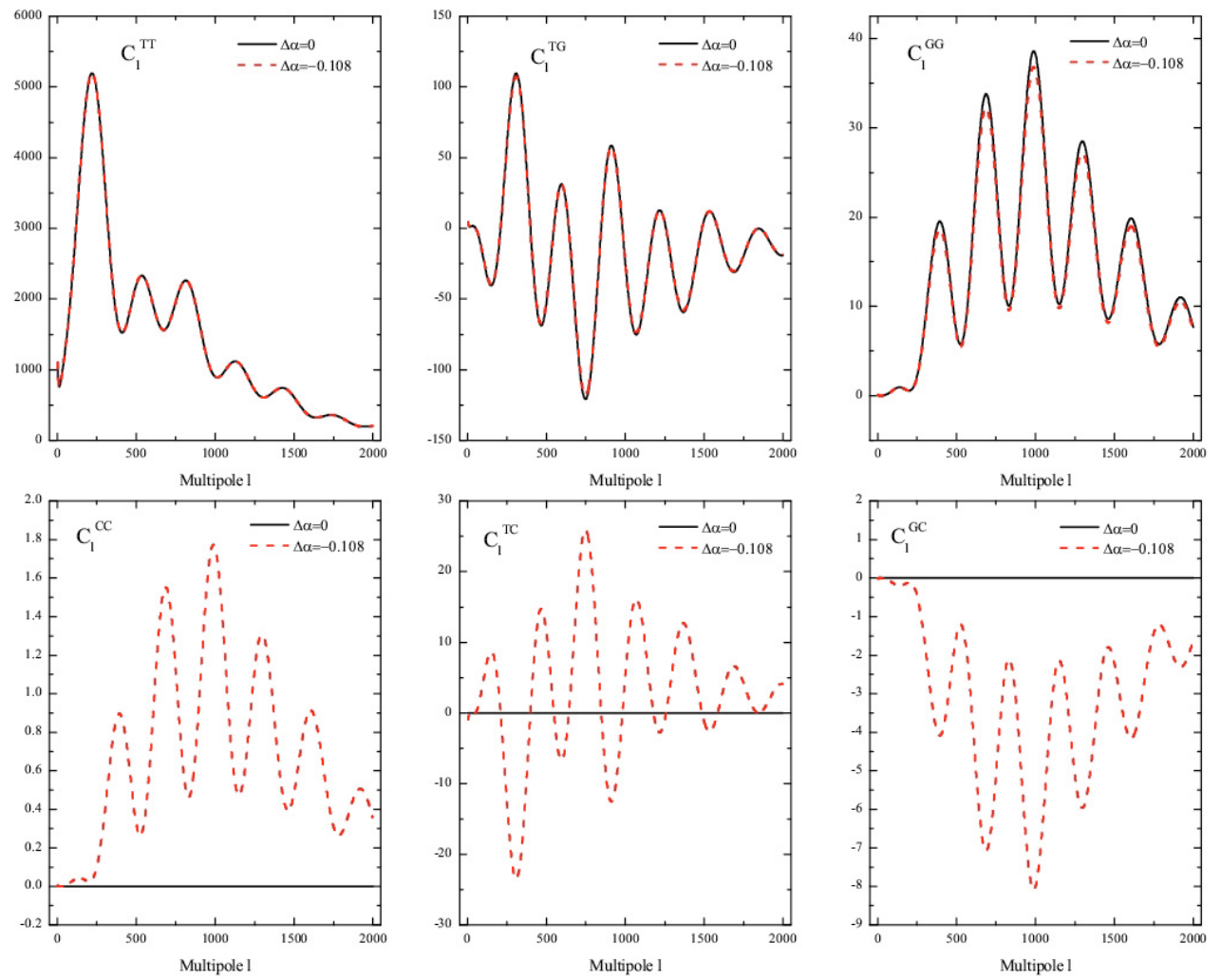

Fig. 2. The effects of the rotation angle $\Delta \alpha$ on the power spectra of TT, TG, GG, CC, TG, and GC. The black solid line is for the case of $\Delta \alpha=0$ and the red dashed line is for $\Delta \alpha=-0.108$.

Table 1. Assumed experimental specifications. The noise parameters $\Delta_{\mathrm{T}}$ and $\Delta_{\mathrm{P}}$ are given in units of $\mu \mathrm{K}$-arcmin.

\begin{tabular}{cccccc}
\hline \hline$f_{\text {sky }}$ & $l_{\max }$ & $(\mathrm{GHz})$ & $\theta_{\text {fwhm }}$ & $\Delta_{\mathrm{T}}$ & $\Delta_{\mathrm{P}}$ \\
\hline 0.65 & 2500 & 100 & $9.5^{\prime}$ & 6.8 & 10.9 \\
& & 143 & $7.1^{\prime}$ & 6.0 & 11.4 \\
& & 217 & $5.0^{\prime}$ & 13.1 & 26.7 \\
\hline
\end{tabular}

The likelihood has been normalized with respect to the maximum likelihood $\chi_{\text {eff }}^{2}=0$, where $\bar{C}_{1}^{\mathrm{XY}}=\hat{C}_{1}^{\mathrm{XY}}$. For more details of this calculation we refer the readers to our previous companion paper Xia et al. (2007). By simulating the data with Planck accuracy, we find that the standard deviation of the rotation angle will be significantly reduced to $\sigma=0.057 \mathrm{deg}$. This result is much more stringent than the current constraint. Assuming the mean value remains unchanged, the cosmological CPT violation will be confirmed around $100 \sigma$ confidence level with the future Planck measurement.

In summary, probing the violation of fundamental symmetries is an important way to search for the new physics beyond the standard model. In this paper we have determined the rotation polarization angle $\Delta \alpha$ with the CMB data from the WMAP3 and BOOMERanG 2003. We find that a nonzero rotation angle of the photons is mildly favored: $\Delta \alpha=-6.2 \pm 3.8 \mathrm{deg}(1 \sigma)$. Our result shows a small violation of the CPT symmetry, albeit not conclusive with the present CMB data. With Planck data our simulations indicate that this type of CPT violation could be confirmed significantly, or CPT symmetry will be verified with high precision.

Acknowledgements. We acknowledge the use of the Legacy Archive for Microwave Background Data Analysis (LAMBDA). Support for LAMBDA is provided by the NASA Office of Space Science. We have performed our numerical analysis on the Shanghai Supercomputer Center (SSC). We are grateful to Eiichiro Komatsu for using of the TC and GC data of WMAP3. We thank Carlo Contaldi, Mingzhe Li, and Hiranya Peiris for helpful discussions. This work is supported in part by National Natural Science Foundation of China under Grant Nos. 90303004, 10533010 and 10675136 and by the Chinese Academy of Science under Grant No. KJCX3-SYW-N2.

\section{References}

Burles, S., Nollett, K. M., Turner, M. S., et al. 2001, ApJ, 552, L1 Cabella, P., Natoli, P., \& Silk, J. 2007, Phys. Rev. D, 76, 123014 Carroll, S. M., \& Field, G. B. 1991, Phys. Rev. D, 43, 3789

Carroll, S. M., Field, G. B., \& Jackiw, R. 1990, Phys. Rev. D, 41, 1231 Davoudiasl, H., Kitano, R., Kribs, C., Murayama, H., \& Steinhardt, P. J. 2004, Phys. Rev. Lett., 93, 201301

De Felice, A., Nasri, S., \& Trodden, M. 2003, Phys. Rev. D, 67, 043509 Feng, B., Li, H., Li, M., \& Zhang, X. M. 2005, Phys. Lett. B, 620, 27

Feng, B., Li, M., Xia, J. Q., Chen, X., \& Zhang, X. M. 2006, Phys. Rev. Lett., 96, 221302

Freedman, W., Madore, B. F., Gibson, B. K., et al. 2001, ApJ, 553, 47

Hinshaw, G., Nolta, M. R., Bennett, C. L., et al. 2007, ApJS, 170, 288

Jarosik, N., Barnes, C., Greason, M. R., et al. 2007, ApJS, 170, 263

Jones, W. C., Ade, P. A. R., Bock, J. J., et al. 2006, ApJ, 647, 823

Kamionkowski, M., Kosowsky, A., \& Stebbins, A. 1997, Phys. Rev. D, 55, 7368 Lewis, A., \& Bridle, S. 2002, Phys. Rev. D, 66, 103511

Li, H., Li, M., \& Zhang, X. M. 2004, Phys. Rev. D, 70, 047302

Li, M., \& Zhang, X. M. 2003, Phys. Lett. B, 573, 20

Li, M., Wang, X. L., Feng, B., \& Zhang, X. M. 2002, Phys. Rev. D, 65, 103511

Li, M., Xia, J. Q., Li, H., \& Zhang, X. M. 2007, Phys. Lett. B, 651, 357

Lue, A., Wang, L. M., \& Kamionkowski, M. 1999, Phys. Rev. Lett., 83, 1506

Montroy, T. E., Ade, P. A. R., Bock, J. J., et al. 2006, ApJ, 647, 813

Page, L., Hinshaw, G., Komatsu, E., et al. 2007, ApJS, 170, 335

Piacentini, F., Ade, P. A. R., Bock, J. J., et al. 2006, ApJ, 647, 833

Scannapieco, E. S., \& Ferreira, P. G. 1997, Phys. Rev. D, 56, 7493

Spergel, D. N., Bean, R., Doré, O., et al. 2007, ApJS, 170, 377

Xia, J. Q., Li, H., Zhao, G.-B., \& Zhang, X. 2007 [arXiv:0708.1111] 